\title{
AVALIAÇÃO DA QUALIDADE DE PAINÉIS COMPENSADOS PRODUZIDOS COM LÂMINAS DE MADEIRA DE Schizolobium amazonicum
}

\author{
Setsuo Iwakiri ${ }^{1}$, Carlos Alberto Vargas ${ }^{2}$, Carlos Frederico Alice Parchen ${ }^{3}$, Christiane Weber ${ }^{4}$, \\ Cilene Cristina Batista ${ }^{5}$, Ernesto Augusto Garbe ${ }^{6}$, Everilton José Cit ${ }^{7}$, José Guilherme Prata $^{1}$ \\ ${ }^{1}$ Eng. Florestal, Dr., Depto. Engenharia e Tecnologia Florestal, UFPR, Curitiba, PR, Brasil - setsuo@ufpr.br; prata@ufpr.br \\ ${ }^{2}$ Designer de Produto, Mestrando em Engenharia Florestal, UFPR, Curitiba, PR, Brasil - sagravargas@ gmail.com \\ ${ }^{3}$ Eng. Civil, M. Sc., Doutorando em Engenharia Florestal, UFPR, Curitiba, PR, Brasil - parchen@ufpr.br \\ ${ }^{4}$ Eng $^{\mathrm{a}}$ Florestal, Mestranda em Engenharia Florestal, UFPR, Curitiba, PR, Brasil - criswef@ yahoo.com.br \\ ${ }^{5}$ Eng $^{\text {a }}$. Ind. Madeireiro, Mestranda em Engenharia Florestal, UFPR, Curitiba, PR, Brasil - cilenecristina@ gamil.com \\ ${ }^{6}$ Eng. Ind. Madeireiro, Mestrando em Engenharia Florestal, UFPR, Curitiba, PR, Brasil - ernestoaugustogarbe@ yahoo.com.br \\ ${ }^{7}$ Eng. Ind. Madeireiro, M.Sc., Doutorando em Engenharia Florestal, UFPR, Curitiba, PR, Brasil - everilton@ yahoo.com.br
}

\section{Resumo}

Esta pesquisa teve por objetivo avaliar a viabilidade de uso de lâminas de madeira de Schizolobium amazonicum (paricá) na produção de painéis compensados. Foram produzidos compensados com cinco lâminas de 2,0 mm de espessura, coladas com resinas ureia-formaldeído (UF) e fenol-formaldeído (FF), com duas diferentes formulações de cola e gramaturas. Foram avaliadas as propriedades de resistência da linha de cola (RLC) e flexão estática (MOE e MOR) paralelo e perpendicular. As diferentes formulações de cola e gramatura não afetaram significativamente os resultados de resistência da linha de cola. Os valores médios de RLC e falhas na madeira atendem aos requisitos da norma EN 314-2 (EUROPEAN COMMITTEE FOR STANDARDIZATION (CEN), 1993). Os resultados de MOE e MOR, sentido paralelo e perpendicular, obtidos para os compensados de paricá foram inferiores em relação aos painéis comerciais de Pinus taeda. Entretanto, a baixa massa específica dos painéis de paricá, entre 0,32 e $0,37 \mathrm{~g} / \mathrm{cm}^{3}$, em relação aos painéis de pínus de $0,53 \mathrm{~g} / \mathrm{cm}^{3}$, não influenciou de forma proporcional na redução dessas propriedades. Os resultados obtidos indicam a viabilidade de uso de lâminas de paricá para fabricação de painéis compensados.

Palavras-chave: Compensados; paricá; lâminas de madeira.

\begin{abstract}
Evaluation of plywood quality manufactured with veneers of Schizolobium amazonicum. This research was developed to evaluate the feasibility to use the veneers of Schizolobium amazonicum (paricá) for plywood manufacture. The plywood was manufactured with five veneers of $2.0 \mathrm{~mm}$ thickness, using ureaformaldehyde and phenol-formaldehyde resins with two different adhesive formulations and amount of glue spread. Were evaluated the properties of glue line shear strength (RLC) and static bending (MOE and MOR) parallel and perpendicular. The different adhesive formulations and amount of glue spread not affected significantly the results of glue line shear strength. The average values of RLC and percentage of wood failures of the Paricá plywood attends the requirements of EN 314-2 (EUROPEAN COMMITTEE FOR STANDARDIZATION (CEN), 1993). The results of MOE and MOR, parallel and perpendicular, obtained to Paricá plywood were lower than the commercial plywood of Pinus taeda. However, the lower density of Paricá plywood $\left(0,32\right.$ to $\left.0,37 \mathrm{~g} / \mathrm{cm}^{3}\right)$ in comparison to pinus plywood $\left(0,53 \mathrm{~g} / \mathrm{cm}^{3}\right)$, not affected significantly to reduction of these properties. The results obtained in this research showed the feasibility of the use of veneer of Paricá for plywood manufacture.

Keywords: Plywood; paricá; veneers.
\end{abstract}

\section{INTRODUÇÃO}

A produção de painéis compensados no Brasil atingiu o patamar máximo de 3,8 milhões de $\mathrm{m}^{3}$ em 2004, sendo 2,43 milhões de $\mathrm{m}^{3}$ de compensado de pínus e 1,38 milhões de $\mathrm{m}^{3}$ de compensados de 
madeira tropical. O compensado é o principal produto de exportação do setor madeireiro brasileiro, cujo volume exportado em 2004 foi da ordem de 3,0 milhões de $\mathrm{m}^{3}$, contribuindo com cerca de 900 milhões de dólares na geração de divisas para o país (ABIMCI, 2007). A contribuição desse segmento em termos econômicos e sociais para o país é altamente significativa, tendo muitos municípios de pequeno porte que dependem economicamente de atividades produtivas que giram em torno de indústrias de compensados.

De acordo com as espécies empregadas na sua produção, os painéis compensados podem ser divididos em dois grupos: compensados de madeiras tropicais e compensados de pínus. Com relação ao compensado de pínus, a sua produção aumentou de forma expressiva a partir de meados da década de 90 , ultrapassando em termos de volume de produção os compensados de madeiras tropicais a partir de 1999 .

Na produção de compensados de madeiras tropicais são utilizadas inúmeras espécies tropicais da Amazônia, tais como amescla (Trattinickia burserifolia), assacu (Hura crepitans), sumaúma (Ceiba pentandra), caucho (Castilloa ulei), faveira (Parkia paraensis), ucuúba (Virola spp.) etc. (ABIMCI, 2007). Entretanto, o impacto ambiental resultante da exploração seletiva de madeiras tropicais tem causado restrições quanto à sua demanda pela parte das comunidades internacionais, sendo que muitos países passaram a exigir certificados de manejo florestal sustentado. Os empresários do setor florestal e madeireiro da Amazônia, preocupados com esse cenário, passaram a investir em alternativas, como implantação de florestas plantadas com espécies nativas da região. Uma dessas espécies é o Schizolobium amazonicum, conhecido como paricá, que demonstrou ser uma excelente alternativa quanto aos aspectos silviculturais, além de produzir madeira com características tecnológicas favoráveis à produção de lâminas e compensados.

Os plantios em escala comercial de paricá (Schizolobium amazonicum Huber ex. Ducke) estão concentrados no estado do Pará, especialmente na região de Paragominas. De acordo com os dados da Sociedade Brasileira de Silvicultura (SBS, 2006), estima-se que a área de plantios seja superior a 50 mil ha. A sua madeira apresenta coloração branca e tem densidade média de $0,40 \mathrm{~g} / \mathrm{cm}^{3}$. O paricá é uma espécie de madeira tropical que apresenta um crescimento acelerado, pode ser implantado em plantios homogêneos ou consorciados e apresenta a vantagem de ser resistente ao ataque de pragas e doenças. A sua produtividade média anual é na faixa de 20 a $30 \mathrm{~m} / \mathrm{ha} / \mathrm{ano}$, estando na mesma faixa de crescimento das espécies de Pinus, que é de 25 a 30 m³/ha/ano, e superior à teca, com 15 a 20 m³/ha/ano (SBS, 2006).

Na produção de compensados, os fatores relacionados à tecnologia devem ser avaliados com muita atenção, principalmente quanto ao processo físico-químico envolvido na colagem de lâminas de madeira (MARRA, 1992). O tipo de resina empregado na colagem deve ser adequado ao ambiente de utilização do painel, podendo ser ureia-formaldeído para ambiente interno e fenol-formaldeído para ambiente externo. A formulação da batida de cola e a gramatura são parâmetros que irão influenciar diretamente na qualidade da colagem e ao mesmo tempo no custo de produção do compensado (BALDWIN, 1995; SELLERS, 1993). Os autores afirmam que a densidade da madeira é um fator importante na definição desses parâmetros, tendo em vista as interações que ocorrem entre a porosidade da madeira e a absorção do adesivo na formação da ligação adesiva entre as lâminas. De acordo com Marra (1992), madeiras de baixa densidade absorvem maior quantidade de adesivo devido à sua maior porosidade, portanto a viscosidade do adesivo deve ser aumentada, para evitar a formação da linha de cola "faminta". Outros fatores, como pH e extrativos presentes na madeira, são também importantes, podendo interferir na cura do adesivo durante o processo de prensagem do painel na prensa quente. Baldwin (1995) afirma que alguns extrativos presentes nas lâminas podem dificultar o processo de vaporização e sua migração de uma linha de cola para outra, e desta para as bordas do painel e posterior liberação para o ambiente externo. Esse processo, sendo muito lento, terá como consequência o aumento da pressão interna de vapor, resultando no "estouro" no momento da abertura da prensa e delaminação do painel.

Este trabalho teve como objetivo avaliar a qualidade de painéis compensados produzidos com lâminas de madeira de paricá, com diferentes formulações de cola e gramaturas.

A pesquisa será desenvolvida para avaliar a viabilidade de uso da madeira de paricá proveniente de plantios florestais na Amazônia para produção de painéis compensados.

\section{MATERIAL E MÉTODOS}

Foram utilizadas nesta pesquisa lâminas de madeira de paricá com densidade média de $0,31 \mathrm{~g} / \mathrm{cm}^{3}$ e espessura de 2.0, coletadas numa indústria laminadora localizada no município de Paragominas, estado do Pará. 
Para a colagem de lâminas, foram utilizadas as resinas ureia-formaldeído (UF) e fenolformaldeído (FF), com teor de sólidos de 65\% e 49\%; pH 8,0 e 11,3; viscosidade Brookfield de $420 \mathrm{cP}$ e $410 \mathrm{cP}$, respectivamente.

Os painéis compensados foram produzidos em escala laboratorial, conforme os planos experimentais apresentados na tabela 1 .

Tabela 1. Plano experimental: resina ureia-formaldeído (UF) e fenol-formaldeído (FF).

Table 1. Experimental chart: urea-formaldehyde and phenol-formaldehyde resin.

\begin{tabular}{lcc}
\hline Tratamento & Formulação & Gramatura $\left(\mathbf{g} / \mathbf{m}^{\mathbf{2}}\right)$ \\
\hline T1 - UF & A & 280 \\
T2 - UF & A & 320 \\
T3 - UF & B & 280 \\
T4 - UF & B & 320 \\
T1 - FF & A & 280 \\
T2 - FF & A & 320 \\
T3 - FF & B & 280 \\
T4 - FF & B & 320 \\
\hline
\end{tabular}

As lâminas de madeira, seccionadas com dimensões de 50 x $50 \mathrm{~cm}$, foram secas em estufa até o teor de umidade médio de $8 \%$. Foram produzidos painéis com cinco lâminas, sendo três por tratamento, utilizando-se resina ureia-formaldeído e fenol-formaldeído, de acordo com as formulações da batida de cola apresentadas na tabela 2 .

Tabela 2. Formulações das batidas de cola.

Table 2. Adhesive formulations.

\begin{tabular}{llccccc}
\hline Resina / formulação & Resina & C. coco & Trigo & Água & Catalisador \\
\hline Ureia-formaldeído & A & 100 & - & 40 & 40 & 1,7 \\
& B & 100 & - & 80 & 80 & 2,1 \\
Fenol-formaldeído & A & 100 & 5 & 5 & 5 & - \\
& B & 100 & 5 & 15 & 15 & - \\
\hline
\end{tabular}

O adesivo foi aplicado manualmente com espátula sobre a superfície das lâminas, de acordo com as gramaturas preestabelecidas de 280 e $320 \mathrm{~g} / \mathrm{m}^{2}$ de superfície dupla. Os painéis foram prensados com pressão específica de $10 \mathrm{kgf} / \mathrm{cm}^{2}$, temperatura de $95^{\circ} \mathrm{C}$ e tempo de prensagem de 8 minutos para colagem com resina ureia-formaldeído, e pressão específica de $10 \mathrm{kgf} / \mathrm{cm}^{2}$, temperatura de $110{ }^{\circ} \mathrm{C}$ e tempo de prensagem de 12 minutos para colagem com resina fenol-formaldeído.

Após o processo de acondicionamento dos painéis na câmara climática com temperatura de $20 \pm 2{ }^{\circ} \mathrm{C}$ e umidade relativa de $65 \pm 5 \%$, foram retirados corpos de prova para realização dos seguintes ensaios em laboratório: resistência da linha de cola aos esforços de cisalhamento (teste seco e fervura) e flexão estática para determinação de módulo de elasticidade (MOE) e módulo de ruptura (MOR) no sentido paralelo e perpendicular. Os ensaios foram realizados de acordo com os procedimentos descritos nas Normas Europeias EN 314 e EN 320, respectivamente.

Os resultados obtidos foram avaliados através da análise de variância e teste de Tukey ao nível de probabilidade de $95 \%$. O delineamento utilizado foi o inteiramente casualizado.

\section{RESULTADOS E DISCUSSÃO}

\section{Painéis colados com resina ureia-formaldeído}

Resistência da linha de cola aos esforços de cisalhamento

Os valores médios de resistência da linha de cola e percentagem de falhas na madeira dos painéis UF, obtidos no teste seco, estão apresentados na tabela 3 .

Os resultados obtidos indicam que não há influência significativa da formulação da batida de cola sobre a resistência da linha de cola dos painéis testados (T1 x T3 e T2 x T4). Com relação à gramatura, para os painéis produzidos com a formulação $\mathrm{B}$, foi constatado um aumento estatisticamente significativo da resistência da linha de cola com o aumento da gramatura de $280 \mathrm{~g} / \mathrm{m}^{2}$ para $320 \mathrm{~g} / \mathrm{m}^{2}$ 
(T3 x T4). Para a formulação A, não foi constatada diferença significativa entre as duas gramaturas de cola, embora em termos de médias absolutas o aumento na gramatura de $280 \mathrm{~g} / \mathrm{m}^{2}$ para $320 \mathrm{~g} / \mathrm{m}^{2}$ tenha contribuído para incremento no valor médio de resistência da linha de cola.

Tabela 3. Resistência da linha de cola ao cisalhamento / teste seco - painéis UF.

Table 3. Glue line shear strength / dry test - UF.

\begin{tabular}{lccc}
\hline Tratamento & Média $(\mathbf{M P a})$ & CV $(\%)$ & FM $(\%)$ \\
\hline T1 - A / 280 & $0,77 \mathrm{AB}$ & 25,73 & 100 \\
T2 - A / 320 & $0,87 \mathrm{AB}$ & 26,67 & 89 \\
T3 - B / 280 & $0,72 \mathrm{~A}$ & 25,73 & 100 \\
T4 - B / 320 & $0,94 \mathrm{~B}$ & 15,49 & 92 \\
\hline
\end{tabular}

$\mathrm{CV}$ : coeficiente de variação; FM: falhas na madeira.

Na comparação com os valores referenciais apresentados na literatura, os resultados obtidos nesta pesquisa estão abaixo dos valores médios encontrados por Iwakiri et al. (2002) para painéis compensados de Pinus taeda produzidos com resina ureia-formaldeído, cujos valores foram na faixa de 1,06 a 1,57 MPa. Bortoleto Jr. obteve, para compensados de Schizolobium parayba (guapuruvu), resistência da linha de cola de 1,51 MPa e 53\% de falhas na madeira. Essas diferenças entre as espécies podem ser atribuídas à baixa densidade da madeira de paricá $\left(0,31 \mathrm{~g} / \mathrm{cm}^{3}\right)$, que contribuiu para maior absorção do adesivo, resultando na redução da espessura da linha de cola e resistência da ligação adesiva entre as lâminas.

Por outro lado, cabe ressaltar que os valores médios de resistência da linha de cola obtidos para os compensados de paricá atendem aos requisitos mínimos da Norma Europeia EN 314-2 (EUROPEAN COMMITTEE FOR STANDARDIZATION (CEN), 1993) para painéis de uso interior, cujos valores são de 0,6 a 1,0 MPa, associados à percentagem de falhas na madeira superior a $40 \%$. Para todos os tratamentos, as percentagens de falhas na madeira foram superiores a $80 \%$, conforme estabelecido pela Norma EN 314-2 (CEN, 1993). De acordo com a referida norma, para $80 \%$ de falhas na madeira a tensão mínima de cisalhamento poderia ser na faixa de 0,2 a $0,4 \mathrm{MPa}$.

Flexão estática paralela

Na tabela 4 estão apresentados os valores médios de massa específica, módulo de elasticidade (MOE) e módulo de ruptura (MOR) paralelo, obtidos para os painéis compensados de paricá.

Tabela 4. Resultados de massa específica e flexão estática paralela - UF.

Table 4. Results of density and static bending (parallel) - UF.

\begin{tabular}{lcccccc}
\hline \multirow{2}{*}{ Tratamento } & \multicolumn{2}{c}{ ME $\left(\mathbf{g} / \mathbf{c m}^{\mathbf{3}}\right)$} & \multicolumn{2}{c}{ MOE $(\mathbf{M P a})$} & \multicolumn{2}{c}{ MOR (MPa) } \\
\cline { 2 - 7 } & Média & $\mathbf{C V ~}(\boldsymbol{\%})$ & Média & $\mathbf{C V}(\%)$ & Média & CV (\%) \\
\hline T1 - A / 280 & 0,371 & 3,69 & $4.234 \mathrm{~B}$ & 2,26 & $33,2 \mathrm{~B}$ & 8,17 \\
$\mathrm{~T} 2$ - A / 320 & 0,345 & 1,18 & $3.880 \mathrm{AB}$ & 5,60 & $24,1 \mathrm{~A}$ & 21,48 \\
$\mathrm{~T} 3$ - B / 280 & 0,335 & 2,99 & $3.607 \mathrm{~A}$ & 6,27 & $23,6 \mathrm{~A}$ & 8,82 \\
$\mathrm{~T} 4$ - B / 320 & 0,343 & 5,38 & $3.444 \mathrm{~A}$ & 11,15 & $23,4 \mathrm{~A}$ & 23,22 \\
\hline
\end{tabular}

ME: massa específica; MOE: módulo de elasticidade; MOR: módulo de ruptura; CV: coeficiente de variação.

Os valores médios da massa específica dos painéis variaram na faixa de 0,335 a $0,371 \mathrm{~g} / \mathrm{cm}^{3}$, sendo esses valores bem inferiores em comparação aos painéis compensados comerciais de Pinus taeda, com média em torno de $0,53 \mathrm{~g} / \mathrm{cm}^{3}$ (ABIMCI, 2002).

As diferentes formulações da batida de cola influenciaram de forma positiva apenas para os painéis produzidos com gramatura de $280 \mathrm{~g} / \mathrm{m}^{2}$ (T1 x T3), em que a formulação A, com maior proporção de resina UF, resultou em maior MOE paralelo. Já para gramatura de $320 \mathrm{~g} / \mathrm{m}^{2}$, não foi constatada diferença estatisticamente significativa entre os painéis produzidos com diferentes formulações (T2 x T4). Não foram constatadas diferenças significativas entre as médias de MOE paralelo para os painéis produzidos com diferentes gramaturas. As mesmas tendências verificadas para o MOE paralelo foram observadas para o MOR paralelo, entre os painéis produzidos com diferentes formulações. Com relação à gramatura, as diferenças significativas foram observadas apenas para os painéis produzidos com formulação A, em que o aumento na gramatura resultou na redução do MOR paralelo. 
Os resultados de MOE e MOR paralelo obtidos para os compensados de paricá foram inferiores em comparação aos valores obtidos por Iwakiri et al. (2001) para painéis de Pinus taeda, cujos valores médios foram respectivamente de 4.330 MPa e 32,0 MPa. Bortoletto Jr. (2003) obteve para painéis compensados produzidos com lâminas de Schizolobium parahyba (guapuruvu), com massa específica de $0,462 \mathrm{~g} / \mathrm{cm}^{3}$, valores de MOE e MOR paralelo de 6.425 e 51,7 MPa, respectivamente. As diferenças verificadas podem ser atribuídas à maior massa específica dos painéis compensados de Pinus taeda de $0,50 \mathrm{~g} / \mathrm{cm}^{3}$ e de Schizolobium parahyba (guapuruvu) de $0,462 \mathrm{~g} / \mathrm{cm}^{3}$, que contribuíram para maiores valores de MOE e MOR em flexão estática.

Com relação aos painéis comerciais de Pinus taeda, os dados apresentados no catálogo técnico da ABIMCI são de 6.890 MPa e 38,1 MPa, respectivamente, para MOE e MOR paralelo, para massa específica dos painéis de $0,53 \mathrm{~g} / \mathrm{cm}^{3}$. Portanto, a densidade da madeira utilizada na manufatura de compensados tem uma influência direta nas propriedades de MOE e MOR em flexão estática.

Flexão estática perpendicular

Na tabela 5 estão apresentados os valores médios de massa específica, módulo de elasticidade (MOE) e módulo de ruptura (MOR) perpendicular, obtidos para os painéis compensados de paricá.

Tabela 5. Resultados de massa específica e flexão estática perpendicular - UF.

Table 5. Results of density and static bending (perpendicular) - UF.

\begin{tabular}{lcccccc}
\hline \multirow{2}{*}{ Tratamento } & \multicolumn{2}{c}{ ME $\left(\mathbf{g} / \mathbf{c m}^{3}\right)$} & \multicolumn{2}{c}{ MOE $(\mathbf{M P a})$} & \multicolumn{2}{c}{ MOR $(\mathbf{M P a})$} \\
\cline { 2 - 7 } & Média & $\mathbf{C V}(\%)$ & Média & $\mathbf{C V}(\%)$ & Média & CV $(\%)$ \\
\hline $\mathrm{T} 1$ - A / 280 & 0,372 & 1,28 & $1.586 \mathrm{~B}$ & 11,60 & $19,1 \mathrm{~B}$ & 21,23 \\
$\mathrm{~T} 2$ - A / 320 & 0,328 & 2,69 & $1.230 \mathrm{~A}$ & 10,76 & $12,4 \mathrm{~A}$ & 23,68 \\
$\mathrm{~T} 3$ - B / 280 & 0,357 & 2,64 & $1.435 \mathrm{AB}$ & 7,04 & $17,9 \mathrm{~B}$ & 14,70 \\
$\mathrm{~T} 4$ - B / 320 & 0,325 & 3,93 & $1.304 \mathrm{~A}$ & 12,71 & $11,2 \mathrm{~A}$ & 14,56 \\
\hline
\end{tabular}

ME: massa específica; MOE: módulo de elasticidade; MOR: módulo de ruptura; CV: coeficiente de variação.

Para estas amostras de painéis, os valores médios da massa específica variaram na faixa de 0,325 a $0,372 \mathrm{~g} / \mathrm{cm}^{3}$.

Tanto para o MOE quanto para o MOR perpendicular, as diferentes formulações da batida de cola não influenciaram de forma estatisticamente significativa nos valores médios dessas propriedades (T1 x T3 e T2 x T4). Por outro lado, para a mesma formulação da batida de cola, os painéis produzidos com a gramatura de $320 \mathrm{~g} / \mathrm{m}^{2}$ apresentaram valores médios de MOE e MOR perpendicular inferiores em relação aos painéis produzidos com gramatura de $280 \mathrm{~g} / \mathrm{m}^{2}$. Esse resultado é importante sob o ponto de vista econômico, devido à possibilidade de redução no consumo de cola.

De acordo com o catálogo técnico da ABIMCI, os valores médios de MOE e MOR perpendicular dos painéis comerciais de Pinus taeda são, respectivamente, de $2.839 \mathrm{MPa}$ e 25,3 MPa, para massa específica de $0,53 \mathrm{~g} / \mathrm{cm}^{3}$. Portanto, os valores obtidos para os painéis de paricá podem ser considerados satisfatórios, tendo em vista sua menor massa específica.

\section{Painéis colados com resina fenol-formaldeído}

Resistência da linha de cola aos esforços de cisalhamento

Os valores médios de resistência da linha de cola e percentagem de falhas na madeira dos painéis FF obtidos no teste de fervura estão apresentados na tabela 6 .

Tabela 6. Resistência da linha de cola ao cisalhamento / teste fervura - FF.

Table 6. Glue line shear strength / boil test - PF.

\begin{tabular}{lccc}
\hline Tratamento & Média (MPa) & CV (\%) & FM (\%) \\
\hline T1 - A / 280 & $0,84 \mathrm{~A}$ & 12,03 & 61 \\
T2 - A / 320 & $0,67 \mathrm{~A}$ & 16,97 & 21 \\
T3 - B / 280 & $0,83 \mathrm{~A}$ & 23,73 & 44 \\
T4 - B / 320 & $0,83 \mathrm{~A}$ & 22,65 & 71 \\
\hline
\end{tabular}

CV: coeficiente de variação; FM: falhas na madeira. 
Não foram constatadas diferenças significativas nos valores de resistência da linha de cola entre os painéis produzidos com diferentes formulações da batida de cola e gramatura. A igualdade estatística entre os resultados obtidos indica a possibilidade de colagem de painéis com a formulação mais econômica (B) e menor gramatura $\left(280 \mathrm{~g} / \mathrm{m}^{2}\right)$.

Com relação aos resultados apresentados na literatura, os valores médios de resistência da linha de cola obtidos nesta pesquisa estão abaixo dos valores médios na faixa de 0,94 a 1,26 MPa, encontrados por Iwakiri et al. (2002) para painéis compensados de Pinus taeda produzidos com resina fenolformaldeído. Bortoletto Jr. (2003) obteve, para painéis compensados fenólicos produzidos com 11 espécies de eucalipto, resistência da linha de cola no teste de fervura na faixa de 1,91 a 2,12 MPa, porém com percentagem de falhas na madeira abaixo de $20 \%$. Pode-se ressaltar que essas diferenças entre as espécies podem ser atribuídas à baixa densidade da madeira de paricá $\left(0,31 \mathrm{~g} / \mathrm{cm}^{3}\right)$, que contribuiu para maior absorção do adesivo, resultando na redução da espessura da linha de cola e resistência da ligação adesiva entre as lâminas. Tanto para a colagem ureica quanto para a fenólica, sugere-se que a viscosidade da batida de cola seja aumentada, para minimizar os problemas de linha de cola faminta, como referenciado por Marra (1992), na colagem de lâminas de madeiras de densidades muito baixas.

$\mathrm{Na}$ avaliação comparativa com as normas, com exceção do tratamento T2, todos os demais tratamentos apresentaram resultados satisfatórios em relação aos requisitos mínimos da Norma Europeia EN 314-2 (CEN, 1993), para painéis de uso exterior, com valores de referências de 0,6 a 1,0 $\mathrm{MPa}$ associados à percentagem de falhas na madeira superior a $40 \%$.

Flexão estática paralela

Na tabela 7 estão apresentados os valores médios de massa específica, módulo de elasticidade (MOE) e módulo de ruptura (MOR) paralelo, obtidos para os painéis compensados de paricá com colagem FF.

Para essas amostras de painéis, os valores médios de massa específica variaram na faixa de 0,349 a $0,394 \mathrm{~g} / \mathrm{cm}^{3}$.

Tabela 7. Resultados de massa específica e flexão estática sentido paralelo - FF.

Table 7. Results of density and static bending (parallel) - PF.

\begin{tabular}{lcccccc}
\hline \multirow{2}{*}{ Tratamento } & \multicolumn{2}{c}{ ME $\left(\mathbf{g} / \mathbf{c m}^{\mathbf{3}}\right)$} & \multicolumn{2}{c}{ MOE (MPa) } & \multicolumn{2}{c}{ MOR (MPa) } \\
\cline { 2 - 7 } & Média & $\mathbf{C V ~}(\%)$ & Média & $\mathbf{C V ~}(\%)$ & Média & CV (\%) \\
\hline T1 - A / 280 & 0,349 & 3,65 & $4.389 \mathrm{~A}$ & 10,43 & $27,4 \mathrm{AB}$ & 8,17 \\
T2 - A / 320 & 0,394 & 2,38 & $5.279 \mathrm{~B}$ & 6,04 & $32,6 \mathrm{~B}$ & 21,48 \\
T3 - B / 280 & 0,353 & 4,01 & $3.965 \mathrm{~A}$ & 10,71 & $21,2 \mathrm{~A}$ & 8,82 \\
T4 - B / 320 & 0,372 & 2,11 & $4.437 \mathrm{~A}$ & 10,05 & $26,3 \mathrm{AB}$ & 23,22 \\
\hline
\end{tabular}

ME: massa específica; MOE: módulo de elasticidade; MOR: módulo de ruptura; CV: coeficiente de variação.

Para painéis produzidos com gramatura de $280 \mathrm{~g} / \mathrm{m}^{2}$, não foram constatadas diferenças estatisticamente significativas para MOE paralelo entre as formulações A e B (T1 x T3). Já para painéis produzidos com gramatura de $320 \mathrm{~g} / \mathrm{m}^{2}$ (T2 x T4), a formulação com maior proporção de resina FF resultou em painéis com maior MOE paralelo. Quanto à influência da gramatura, para os painéis produzidos com formulação A o aumento na gramatura resultou em média estatisticamente superior de MOE paralelo. Entre os painéis produzidos com formulação B não foi constatada diferença significativa, entretanto, em termos de médias absolutas, houve uma tendência de aumento no MOE paralelo com aumento na gramatura.

Com relação ao MOR paralelo, não foram constatadas diferenças estatisticamente significativas entre os painéis produzidos com diferentes formulações da batida de cola e diferentes gramaturas.

Os resultados de MOE e MOR paralelo obtidos para os compensados FF de paricá foram inferiores em comparação aos valores obtidos por Iwakiri et al. (2001) para painéis de Pinus taeda, cujos valores médios foram, respectivamente, de 5.800 MPa e de 58,4 MPa. As diferenças observadas podem ser atribuídas à menor massa específica dos painéis de paricá $\left(0,349\right.$ a $\left.0,394 \mathrm{~g} / \mathrm{cm}^{3}\right)$ em relação aos painéis de Pinus taeda $\left(0,50 \mathrm{~g} / \mathrm{cm}^{3}\right)$. Com relação aos painéis comerciais de Pinus taeda, os dados apresentados no catálogo técnico da ABIMCI são de $6.890 \mathrm{MPa}$ e 38,1 MPa, respectivamente, para o MOE e MOR paralelo, para massa específica dos painéis de $0,53 \mathrm{~g} / \mathrm{cm}^{3}$. 
Flexão estática perpendicular

Na tabela 8 estão apresentados os valores médios de massa específica, módulo de elasticidade (MOE) e módulo de ruptura (MOR) perpendicular, obtidos para os painéis compensados de paricá com colagem FF.

Tabela 8. Resultados de massa específica e flexão estática perpendicular - FF.

Table 8. Results of density and static bending (perpendicular) - PF.

\begin{tabular}{lcccccc}
\hline \multirow{2}{*}{ Tratamento } & \multicolumn{2}{c}{ ME $\left(\mathbf{g} / \mathbf{c m}^{3}\right)$} & \multicolumn{2}{c}{ MOE $(\mathbf{M P a})$} & \multicolumn{2}{c}{ MOR $(\mathbf{M P a})$} \\
\cline { 2 - 7 } & Média & $\mathbf{C V}(\boldsymbol{\%})$ & Média & $\mathbf{C V}(\%)$ & Média & CV $(\%)$ \\
\hline T1 - A / 280 & 0,358 & 0,80 & $1.330 \mathrm{~A}$ & 9,23 & $14,6 \mathrm{AB}$ & 23,15 \\
$\mathrm{~T} 2$ - A / 320 & 0,375 & 3,35 & $1.623 \mathrm{~B}$ & 15,30 & $17,1 \mathrm{~B}$ & 16,59 \\
$\mathrm{~T} 3$ - B / 280 & 0,352 & 2,27 & $1.182 \mathrm{~A}$ & 5,04 & $14,5 \mathrm{AB}$ & 8,63 \\
$\mathrm{~T} 4$ - B / 320 & 0,345 & 2,69 & $1.165 \mathrm{~A}$ & 4,06 & $12,1 \mathrm{~A}$ & 13,99 \\
\hline
\end{tabular}

ME: massa específica; MOE: módulo de elasticidade; MOR: módulo de ruptura; CV: coeficiente de variação.

Para estas amostras de painéis, os valores médios de massa específica variaram na faixa de 0,345 a $0,375 \mathrm{~g} / \mathrm{cm}^{3}$.

Para tratamentos com gramatura de $280 \mathrm{~g} / \mathrm{m}^{2}$ não foi constatada diferença estatisticamente significativa para o MOE perpendicular entre os painéis produzidos com a formulação A e B (T1 x T3). Para a gramatura de $320 \mathrm{~g} / \mathrm{m}^{2}$, os painéis produzidos com formulação A, com maior proporção de resina FF, apresentaram valor médio de MOE perpendicular estatisticamente superior em comparação aos painéis produzidos com formulação B (T2 x T4). Com relação aos efeitos da gramatura, para formulação A, os painéis produzidos com gramatura de $320 \mathrm{~g} / \mathrm{m}^{2}$ apresentaram valor médio de MOE perpendicular estatisticamente superior em comparação aos painéis produzidos com gramatura de $280 \mathrm{~g} / \mathrm{m}^{2}$ (T1 x T2). Para a formulação B não foi constatada diferença significativa entre as diferentes gramaturas (T3 x T4).

Os resultados dos efeitos da formulação da batida de cola obtidos para o MOR perpendicular, indicam as mesmas tendências observadas para o MOE perpendicular. Com relação aos efeitos da gramatura não foram constatadas diferenças estatisticamente significativas entre os painéis produzidos com $280 \mathrm{~g} / \mathrm{m}^{2}$ e $320 \mathrm{~g} / \mathrm{m}^{2}$.

Os resultados de MOE e MOR perpendicular obtidos para compensados de paricá foram inferiores em comparação aos painéis comerciais de Pinus taeda, cujos valores são, respectivamente, de 2.839 MPa e 25,3 Mpa. Essas diferenças podem ser atribuídas à menor massa específica dos painéis de paricá em relação à massa específica dos painéis comerciais de Pinus taeda de $0,53 \mathrm{~g} / \mathrm{cm}^{3}$.

\section{CONCLUSÕES}

Com base nos resultados obtidos nesta pesquisa, as seguintes conclusões podem ser apresentadas:

- Com exceção dos painéis UF produzidos com formulação B, não foram constatadas diferenças significativas dos efeitos da formulação da batida de cola e da gramatura sobre a resistência da linha de cola dos painéis UF e FF. Todos os resultados obtidos atingiram os requisitos mínimos da Norma Europeia EN 314-2 (CEN, 1993).

- Os painéis compensados de paricá apresentaram baixa massa específica, o que contribuiu para redução nos resultados de MOE e MOR paralelo e perpendicular.

- Os painéis produzidos com formulação A apresentaram maiores valores de MOE e MOR para gramatura de $280 \mathrm{~g} / \mathrm{m}^{2}$ (UF) e $320 \mathrm{~g} / \mathrm{m}^{2}$ (FF).

- A análise das diferentes gramaturas demonstrou uma tendência de redução nos valores de MOE e MOR, paralelo e perpendicular, para os painéis UF, com o aumento na gramatura de $280 \mathrm{~g} / \mathrm{m}^{2}$ para $320 \mathrm{~g} / \mathrm{m}^{2}$. Para os painéis FF, o aumento na gramatura resultou em maiores valores médios de MOE e MOR, paralelo e perpendicular, com exceção para o MOR perpendicular com formulação B.

- Os resultados das propriedades avaliadas indicam a viabilidade de uso de lâminas de madeira de paricá provenientes de plantios florestais na Amazônia na produção de painéis compensados.

\section{AGRADECIMENTOS}

Os autores expressam seus agradecimentos às empresas Selectas S.A. e Hexion Química Indústria e Comércio Ltda., pelas doações de lâminas de madeiras de paricá e resinas UF e FF utilizadas nesta pesquisa.

FLORESTA, Curitiba, PR, v. 41, n. 3, p. 451-458, jul./set. 2011.

Iwakiri, S. et al. 


\section{REFERÊNCIAS}

ASSOCIAÇÃO BRASILEIRA DA INDÚSTRIA DA MADEIRA PROCESSADA MECANICAMENTE (ABIMCI). Catálogo técnico de compensados de pinus. Curitiba, 2002. 20 p.

. Estudo setorial. Curitiba, 2007. 40 p.

BALDWIN, R. F. Plywood and veneer-based products: manufacturing practices. San Francisco: Miller Freeman, 1993. $388 \mathrm{p}$.

BORTOLETTO JR., G. Produção de lâminas e manufatura de compensados a partir da madeira de guapuruvu (Schizolobium parahyba Blake.) proveniente de um plantio misto de espécies nativas. Cerne, v. 8 , n. 2 , p. $1-16,2002$.

Produção de compensados com 11 espécies do gênero Eucalyptus, avaliação das suas propriedades físico-mecânicas e indicações para utilização. Scientia Forestalis, n. 63, p. 65 - 78, jun. 2003.

EUROPEAN COMMITTEE FOR STANDARDIZATION (CEN) EN 314-2: 1993: plywood determination of shear bonding strength. 1993.

IWAKIRI, S.; OLANDOSKI, D. P.; LEONHARDT, G.; BRAND, M. A. Produção de chapas de madeira compensada de cinco espécies de pinus tropicais. Ciência Florestal, Santa Maria, v. 11, n. 2, p. 71 - 77 , 2001.

IWAKIRI, S.; DEL MENEZZI, C. H. S.; LAROCA, C.; VENSON, I.; MATOSKI, S. S. Produção de compensados de Pinus taeda e Pinus oocarpa com resina fenol-formaldeído. Cerne, Lavras, v. 11, n. 2, p. 92 - 97, 2002.

IWAKIRI, S.; SILVA, J. C.; SILVA, J. R. M.; ALVES, C. R.; PUEHRINGER, C. A. Produção de compensados de Pinus taeda L. E. e Pinus oocarpa Schiede com diferentes formulações de adesivo ureiaformaldeído. Árvore, Viçosa, v. 26, n. 3, p. 371 - 375, 2002.

MARRA, A. A. Technology of wood bonding: principles in practice. New York: Van Nostrand Reinhold, 1992. 453 p.

SELLERS, T. Plywood and adhesive technology. New York: Marcel Dekker, 1993. 661 p.

SOCIEDADE BRASILEIRA DE SILVICULTURA (SBS). Fatos e números do Brasil florestal - 2006. 2007. Disponível em: <www.sbs.org.br〉. Acesso em: 12/11/2009. 